Syntactic relations in the construction of heritage space. Domestic space organization in the vernacular architecture of San Pedro de Atacama
PALABRAS CLAVE - SAN PEDRO DE ATACAMA ARPUITECTURA PATRIMONIAL · ASENTAMIENTOS ARPUEOLÓCICOS - UNIDAD DOMÉSTICA - VIVIENDA TÍPICA KEYWORDS - SAN PEDRO DE ATACAMA - HERITACE ARCHITECTURE · SETTLEMENT ARCHAEOLOGY · DOMESTIC UNIT - TYPICAL HOUSINC
RESUMEN

Este trabajo se estructura sobre la discusión y el análisis de las relaciones sintácticas en la arquitectura vernácula y patrimonial del territorio atacameño, en relación a su unidad doméstica. En una primera aproximación, a través de la revisión de textos arqueológicos, relacionando algunos asentamientos prehispánicos del período formativo. Y en segunda instancia, a través del análisis de la vivienda típica atacameña, de alto valor patrimonial que se consolidaría a fines del siglo XIX e inicios de siglo $X X$ en el oasis de San Pedro de Atacama. El enfoque de estas temporalidades, tan distantes, se realiza a través del reconocimiento de algunos aspectos jerárquicos que, como relaciones inmateriales, organizan el espacio construido a través de aquellas manifestaciones humanas, enraizadas en lo más profundo del entendimiento de cómo habitar estos territorios.

\section{ABSTRACT}

The following work is based on the discussion and analysis of syntactic relations of the vernacular heritage and architecture of the Atacama territory, regarding its domestic unit. At first, this research was carried out through the review of archaeological texts, linking together the formative period of some pre-Hispanic settlements. subsequently it was reviewed through the analysis of the typical it Atacameños dwelling of high heritage value, which were strengthened at the end of the 19th and the beginning of the twentieth century in San Pedro de Atacama oases. The approach of these distant temporaities is caried out through the recogition of some hierarchical aspects that, as immaterial relations, organize the space built based on those human manifestations, which in turn are rooted in the deepest comprehension of how to inhabit these territories.

\title{
La organización del espacio doméstico en la arquitectura vernácula de San Pedro de Atacama
}

\author{
Relaciones sintácticas en la construcción del espacio \\ patrimonial
}

MG. víCTOR ROJAS TABILO · ETSAB, Universidad Politécnica de Cataluña, Barcelona · vrojast@gmail.com

Fecha de recepción: 15 de marzo 2019 . Fecha de aceptación: 30 de abril 2019

\section{INTRODUCCIÓN}

El oasis de San Pedro de Atacama destaca tanto por su legado arqueológico, etnográfico e histórico, como también por el reconocimiento de su arquitectura patrimonial, declarado "Zona Típica" en 1980. En términos demográficos corresponde al poblado más importante de los localizados en la cuenca del salar de Atacama.

Dentro de los procesos necesarios para el reconocimiento de su arquitectura típica la revisión de la unidad doméstica, como unidad de análisis, ha sido fundamental para entender el panorama arquitectónico de estas zonas patrimoniales. La unidad doméstica será comprendida aquí como el conjunto arquitectónico básico o unidad funcional mínima que consolidan las primeras comunidades sedentarias para permitir su subsistencia y dar lugar a sus actividades domésticas. Wilk y Ashmore (1988) plantean que la conformación de más de una unidad doméstica o household cluster correspondería a los niveles de integración de distintos componentes sociales y agentes productivos determinados, contextualizando a estos espacios dentro de una estructura mayor en un ámbito territorial determinado, considerándolas unidades altamente interiorizadas y autónomas, ya que en ellas se realizarían las actividades domésticas más básicas en una sociedad.
Hasta ahora, un amplio abanico de estudios académicos nos otorga las pautas necesarias para aproximarnos a su entendimiento en San Pedro de Atacama. Sin embargo, aquellos análisis realizados en un horizonte temporal más extenso, incluyendo el patrimonio arqueológico, desde nuestra disciplina aún son escasos.

Por otra parte, el estudio referido a la vivienda típica patrimonial, que ha perdurado hasta nuestros días en los oasis de San Pedro de Atacama, se ha centrado fundamentalmente en la revisión y descripción de sus aspectos constructivos (materialidades y sistemas constructivos). Sin duda, la materia es el elemento angular de toda arquitectura $y$, por lo tanto, el aspecto más importante en su estudio y análisis, sin embargo, las pocas referencias respecto a las relaciones sintácticas también serían fundamentales, sobre todo, si las entendemos como recursos teóricos que nos permitan comprender los sistemas de ordenamiento jerárquico de la tradición arquitectónica local.

En dicho contexto, el objetivo de este artículo es generar las lecturas necesarias que nos aproximen a entender el orden compositivo que subyace en la arquitectura doméstica y patrimonial en el territorio de estudio. Entendiéndolas como un complemento a los estudios ya realizados desde una perspectiva, hasta el momento, poco estudiada. Dichas 
1. A. Vista área de San Pedro de Atacama. Imagen: Haroldo Horta. B. Ubicación de los casos referenciados. (1) As. arqueológico Ghatchi2C, (2) As. arqueológico Ghatchi2B, (3) As. arqueológico Calar, (4) As. arqueológico Tulor, (5), Casa Sra. Iris Hidalgo, (6) Casa de los Ancianos (7) Casa del Pukara 1, (8) Casa Sr. Tijerina, (9) Casa del Pukara 2, (10) Casa familia León. Imagen satelital, San Pedro de Atacama, Chile. SASPlanet.

(14)

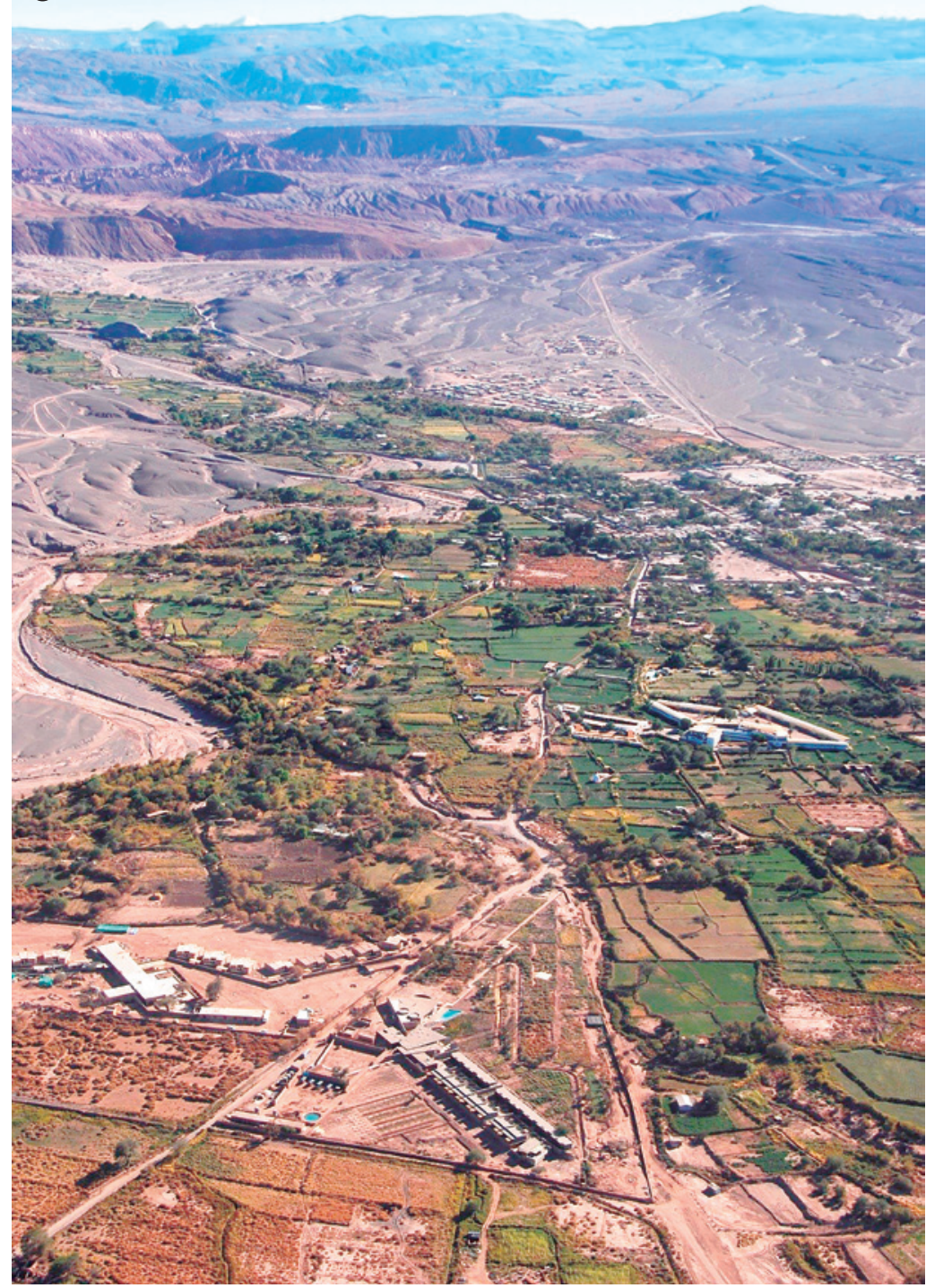

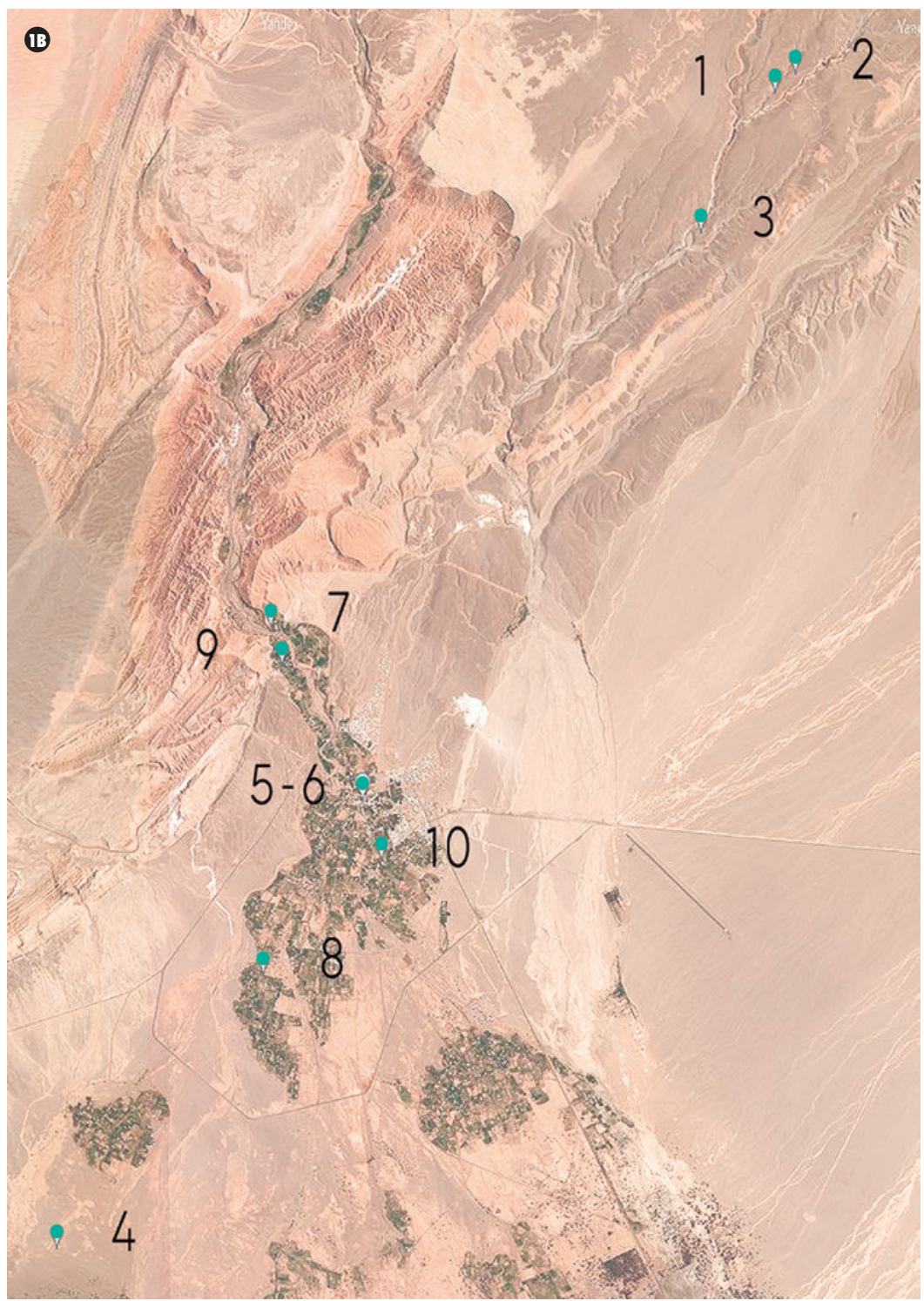

relaciones, además, pueden ser entendidas como recursos teóricos al servicio de los procesos proyectuales de aquella "arquitectura nueva" que siendo contemporánea recoja estos órdenes materiales e inmateriales propiamente locales al intervenir dentro de este contexto patrimonial (FIGURA 1).

La metodología utilizada consistió en la revisión de las aportaciones, análisis y descripciones, realizadas desde el ámbito arqueológico de tres de los asentamientos prehispánicos más documentados del período formativo que, en correspondencia de sus temporalidades, son referentes de una primera arquitectura de carácter doméstico (horizonte temporal lejano); desde un punto de vista general, el período formativo ha sido concebido como el momento en que las sociedades arcaicas, de tradición cazadora recolectora, incorporan e implementan estrategias económicas que permitirían la generación de cierto nivel de acumulación o producción excedentaria (Willey y Philips 1958; Rowe 1962; Lumbreras 1981; Adán y Urbina 2007).

Posteriormente, se revisaron seis casos de aquella arquitectura doméstica, considerada típica y patrimonial de los oasis de San Pedro de Atacama, de fines del siglo XIX e inicios 
de siglo XX (horizonte temporal temprano). Si bien, ambas situaciones corresponden a dos etapas temporales completamente distantes, son representativas de una arquitectura propiamente local que posee relaciones particulares en la organización de sus espacios construidos (relaciones sintácticas) surgidas desde el conocimiento profundo de las sociedades que habitaron este territorio.

\section{RELACIONES SINTÁCTICAS EN LA UNIDAD DOMÉSTICA DE LOS PERÍODOS FORMATIVOS, ASENTAMIENTOS PREHISPÁNICOS}

La arquitectura doméstica que surge en períodos formativos debe ser entendida dentro de un marco temporal bastante amplio, donde las sociedades arcaicas de cazadores recolectores transitarán desde asentamientos esporádicos (refugios y cotos de caza) a construcciones cada vez más permanentes y consolidadas. Dicho evento estaría en estrecha relación con el proceso que significó la domesticación de auquénidos y el desarrollo de una insipiente agricultura, dando paso a una organización del espacio construido cada vez más complejo y consolidado.

La aproximación al tema se realiza a través de las investigaciones arqueológicas efectuadas en los sitios denominados Ghatchi2C y Calar. Sitios realmente interesantes para el estudio de la arquitectura vernácula, más aún, si consideramos que son zonas ampliamente documentadas y estudiadas desde la arqueología (Le Paige, 1963-64; Leonor Adán, 2007; Pimentel, 2008; Agüero Uribe, 2011 ), encontrándose en ellas una gran variabilidad de patrones organizativos en fases temporales tempranas.

Ghatchi 2C (FIGURA 2), en relación a lo expuesto por Adán y Urbina (2007), corresponde a un asentamiento de patrón disperso, ubicado en las laderas de quebradas del rio Vilama, aproximadamente a $10 \mathrm{~km}$ hacia el noroeste de la localidad de San Pedro de Atacama con al
2. A. Levantamiento topográfico Ghatchi-2C. Proyecto FONDECYT 1030931. Agüero y Uribe (2011). Estudios Atacameños: Arqueología y Antropología Surandinas № 34. B. Imagen satelital Asentamiento Ghatchi2C. Fuente; SASPlanet (2019)
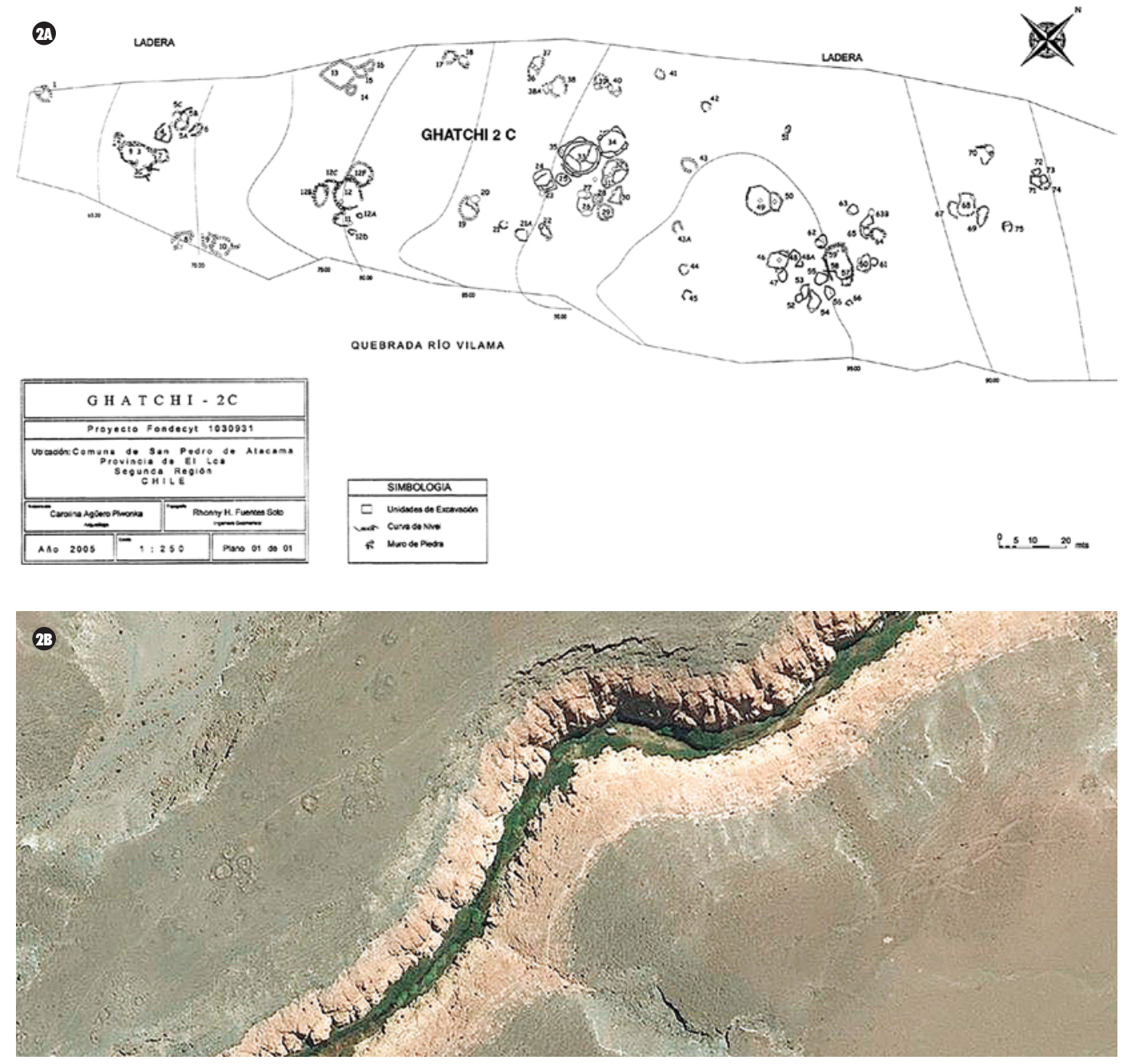

menos dos componentes arquitectónicos, uno arcaico (4000-3350 a. C.) y otro formativo temprano (1200-350 a. C.).

De las caracterizaciones realizadas al sitio, Agüero y Uribe (2011) mencionan respecto a la tipología de las construcciones existentes: "esta variabilidad muestra dos patrones constructivos y momentos ocupacionales. Uno más temprano con estructuras irregulares y de planta circular; y otro más tardío, probablemente transicional y formativo que desarrolla el patrón recinto mayor y menores asociados en distribución radial" (p. 56).
En estos períodos de transición, la centralización de los espacios construidos estaría en función de aquellos primeros corrales, relacionando la importancia que significaría el proceso de aprendizaje de la domesticación de auquénidos y el surgimiento de nuevos mecanismos sociales de integración y participación comunitaria en la obtención de recursos alimentarios, textiles y de intercambio.

Calar (FIGURA 3) situado temporalmente entre el formativo medio (350 a. C. - 100 d. C.) y el formativo tardío (100 a. C. - 500 d. C.) es un referente de los estadios más tardíos del mismo 
3. A. Planta de la aldea de Calar. Modificado de Orellana (1988-1989). Estudios Atacameños: Arqueología y Antropología Surandinas №34. B. Imagen satelital Asentamiento Calar. Fuente: SASPlanet (2019).
(3)
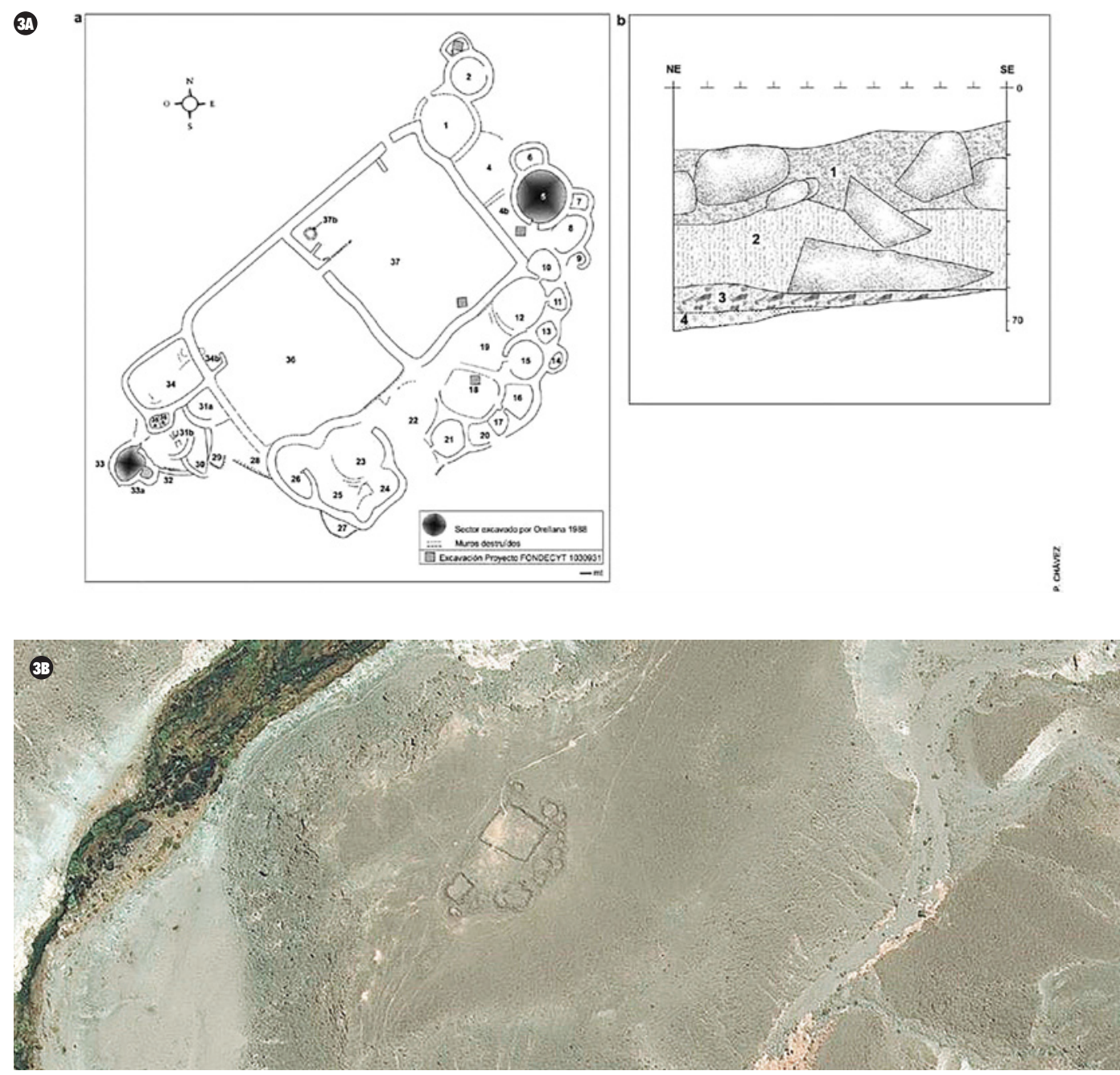

proceso de consolidación, se conformaría a partir de estructuras construidas y reconstruidas en fases temporales sumamente prolongadas, que irían desde el formativo medio al tardío. Se ordena en disposición radial, con estructuras concéntricamente distribuidas que van desde las construcciones mayores a las menores. Esta configuración evidencia claras diferencias respecto a sus usos específicos, como también, referencia distintos grados de privacidad y de exposición en relación a las actividades públicas-comunitarias y privadas-íntimas establecidas por la comunidad.
En este contexto, el conjunto arquitectónico presenta una gran variabilidad de formas y espacialidades, incluidos los interespacios o espacialidades de transición, que otorgarían distintos grados de privacidad en los recintos del conjunto arquitectónico. Estos espacios intermedios cobrarían relevancia protagónica en los asentamientos aldeanos que surgirían en este período en los oasis de San Pedro de Atacama.

Respecto a los momentos formativos, el asentamiento está menos afectado en el sector norte, en el cual se instalan los conjuntos anteriores. La sección sur, en cambio, estuvo más afectada por procesos de reconstrucción, habiendo allí un conjunto de una estancia con corral, "chiquero" o "enfermería" y habitación, donde se adicionaría la segunda "cancha" más tardíamente (Agüero \& Uribe, 2011 , pp. 64, 65).

En términos funcionales la documentación arqueológica nos aporta algunos matices de los posibles usos de los recintos; Respecto a los menores, "el conjunto cerámico indica la existencia de recintos habitacionales y contextos primarios, usados de manera exclusiva durante el Formativo, realizándose labores domésticas (p.e., preparación, almacenaje de alimentos y consumo de líquidos)" (Agüero \& Uribe, 2011, p. 65).; Y respecto a las canchas, "referido a la similitud formal de las estructuras tipo 'plazas' o 'patios' con los corrales. (...) nos remite de forma paradigmática al sustento de las sociedades formativas, con el surgimiento de la acumulación y de la producción excedentaria" (Adán \& Urbina, 2007, p. 26).

El asentamiento de Tulor-1 (FIgura 4) situado temporalmente entre el formativo medio (350 a. C. - 100 d. C.) y el formativo tardío (100 a. C. - 500 d. C.), alcanzará un nivel tal de complejidad que se vincula a órdenes propiamente aldeanos, se conforma a partir de un número significativo de unidades domésticas articulando algunas actividades en niveles propiamente urbanos. En este período los espacios intermedios adquieren una relevancia fundamental en el modo de vida y la arquitectura, así lo demuestra el análisis realizado por Adán y Urbina (2007) estableciendo que esta tipología corresponde al $39,6 \%$ del total de los recintos de Tulor-1, siendo el mayor porcentaje de los recintos catalogados. Se describen como espacialidades sumamente prácticas para la demanda espacial requerida en asentamientos conglomerados de altos niveles demográficos, desarrollándose en ellos actividades principalmente destinadas al trabajo comunitario de participación e integración social (FIGURA 4). 
4. A y B. Dibujo de la planta de la aldea Tulor-1. Modificado de Lagostera y colaboradores (1984). Estudios Atacameños: Arqueología y Antropología Surandinas $N^{\circ} 34$.
。
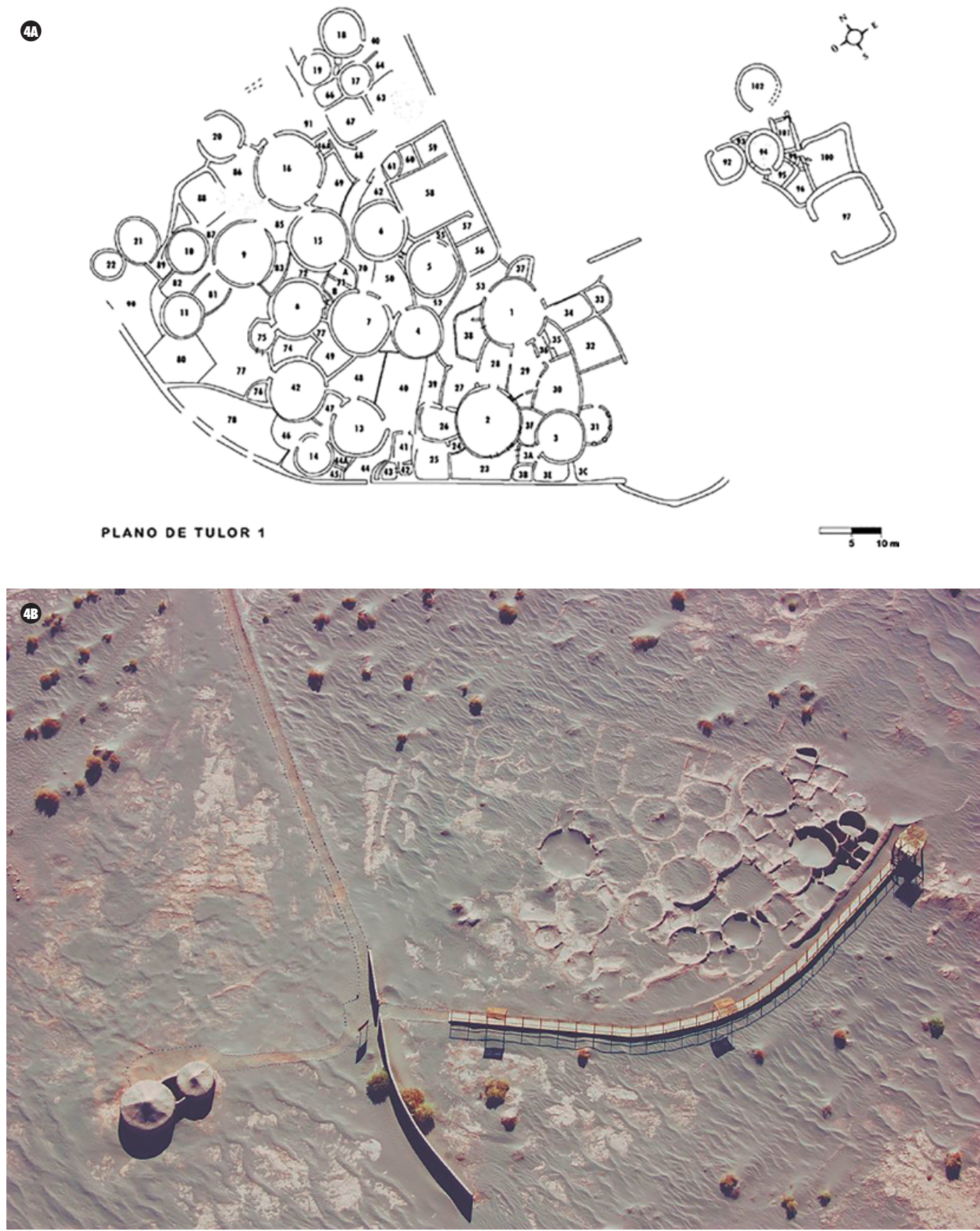

PLANO DE TULOR 1 una horticultura de campos inundados en las en los componentes tardíos de Tulan y Puripica un predominio de las prácticas de caza y pastoreo (Núñez, 2005) (Adán \& Urbina, 2007, p. 10).

En la Figura 5 se exponen esquemáticamente los asentamientos referenciados, respetando la morfología de sus formas en planta, a una misma escala, y diferenciando el tamaño de los recintos. Este ejercicio nos permite contrastar la distribución y organización de las construcciones, como también, la forma y el tamaño de las espacialidades que lo componen. Los gráficos que acompañan a los asentamientos indican la cantidad de recintos en relación a sus tamaños.

En referencia a las plantas construidas, se observa un desarrollo que transitará desde las formas irregulares y orgánicas, en aquellos períodos arcaicos y formativos tempranos, hacia formas geométricas más regulares en períodos posteriores. Rectangulares para las "canchas o plazas" y circulares en la mayoría de los recintos medios y menores.

La organización de las construcciones cambiará progresivamente desde una configuración de patrón disperso, de evidente carácter esporádico, a modelos cada vez más nucleados y centralizados en torno a espacialidades de mayores dimensiones vinculadas a los "corrales y/o plazas", en algunas de los cuales incluso se aprecia una mejor factura que en el resto de las construcciones. En referencia al asentamiento de Ghatchi 2C. Adán y Urbina (2011) al respecto mencionan, "se observan paramentos de hilada simple en un $65 \%$ de la muestra con una significativa ocurrencia de muros dobles y dobles con relleno que alcanzan cerca de un $20 \%$ empleándose en la edificación de los recintos centrales" (pp. 21-22).

Lo que implica una mayor selectividad de los materiales utilizados, como también, una mayor inversión de trabajo y tiempo en su construcción, reafirmando su condición de inmediaciones del río; mientras que en Calary
Es probable que Tulor haya surgido luego de la existencia de unidades menores discretas sobre las que Tulor-1 se habría desarrollado hasta transformarse en aldea comprometiendo los momentos medios y tardíos del período. (...) Lo anterior plantea la coexistencia de prácticas económicas diferenciadas: en Tulor-1 basada en los productos de recolección y en 
5. Esquema y gráfico de los asentamientos: (A) Ghatchi $2 \mathrm{C}$, (B) Ghatchi $2 B(C)$ Calar, (D) Tulor-1. Elaboración propia (2019).
(3)
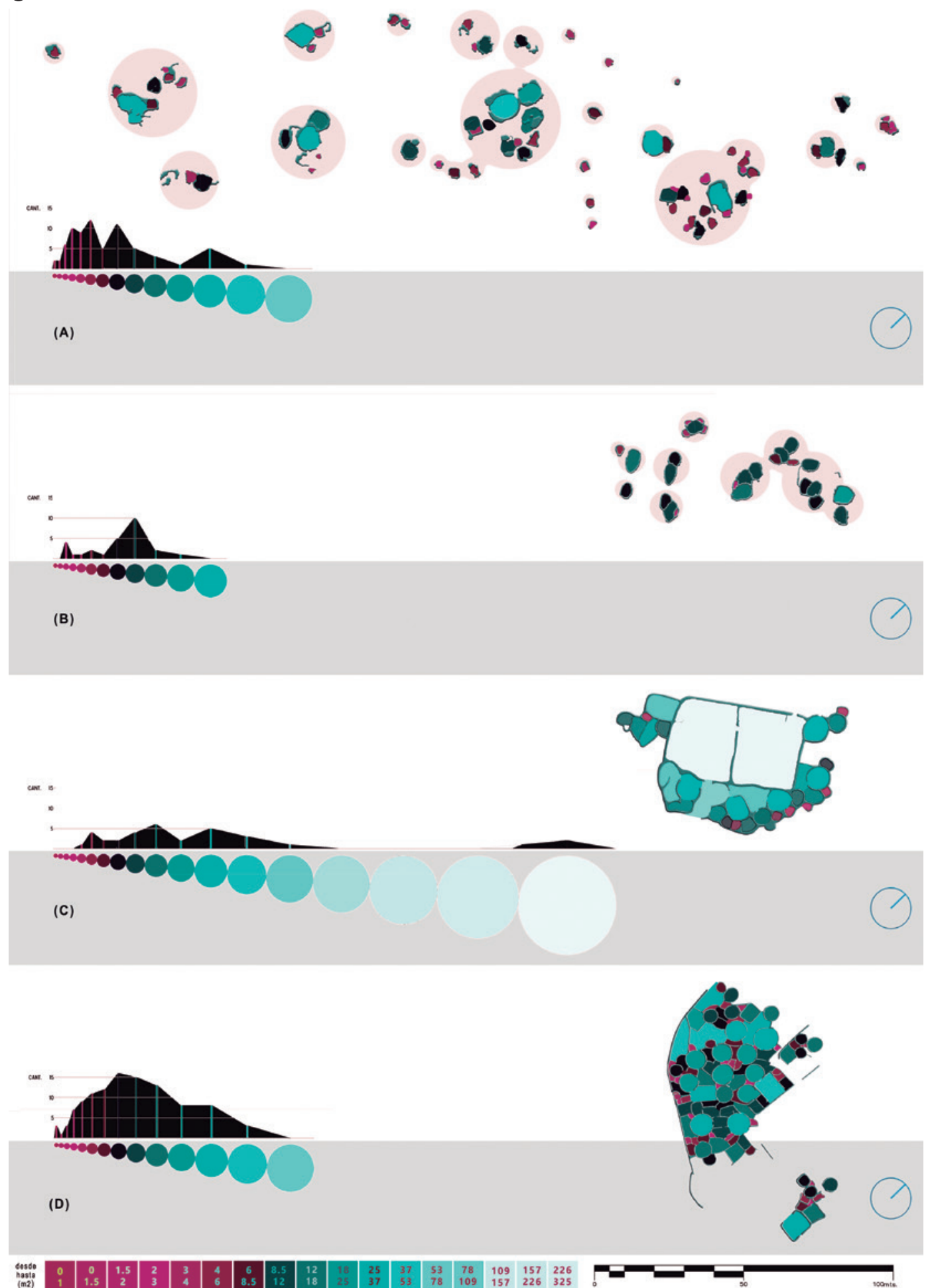

espacialidad fundamental que constituiría el núcleo de estas comunidades, quienes articularían sus construcciones en torno a estos corrales/plazas que representan y simbolizan la estabilidad de los recursos, la acumulación de lo excedentario, y lo público del trabajo comunitario.

Si bien está clara la imposibilidad de asignar monofuncionalmente el uso de las estructuras construidas como lo exponen Adán y Urbina (2007), los estudios de Agüero y Mauricio Uribe (201 1), refiriéndose Ghatchi2C, han demostrado que algunas de las construcciones menores tendrían usos bastante específicos, puntualizando dos de los recintos excavados; un primer recinto, utilizado para la cocción y consumición de alimentos; y un segundo, correspondiente a un montículo exterior adyacente a una de las construcciones, destinado para la acumulación de desechos domésticos. Dicha variabilidad funcional podría ser un indicador probable de la transición a construcciones con formas y dimensiones cada vez más específicas, como las surgidas en los asentamientos del formativo medio y tardío como es el caso de Calar y Tulor-1.

En relación al gráfico expuesto que acompaña su esquema, el asentamiento de Calar presenta una configuración de recintos que podemos agrupar en cuatro magnitudes claramente diferenciadas; dos espacialidades rectangulares de grandes dimensiones al centro, "plazascorrales" de carácter públicas; espacios medios-mayores, adosados a los muros de las plazas antes referidas, probablemente "corrales y chiqueros", en relación a la correspondencia de sus dimensiones con los corrales presentes en las etapas anteriores (p.ej. Ghatchi 2B y $2 \mathrm{C}$ ); espacios medios-menores los cuales, en relación a su tamaño, serían susceptibles de ser cubiertos con estructuras simples, probablemente de resguardo o habitacionales de carácter privado; espacios pequeños no habitables, vinculados a los recintos antes referidos, cuya función probable fuese la de almacenaje. 
En esta complejidad se inauguran las espacialidades de intermediación "interrecintos", espacios que en principio parecen ser residuales resultantes de la adhesión y/o proximidad de las construcciones principales, se consolidarían gradualmente a medida que el patrón de asentamiento cambiaba desde el disperso al aglutinado. En relación a lo expuesto sobre el asentamiento de Tulor-1, en estos espacios se exteriorizarían ámbitos privados e interiorizan ámbitos públicos generando distintos lugares de interacción que, como espacios construidos, conllevan a relaciones nuevas y diferenciadas entre la comunidad como grupo y el espacio comunitario.

\section{RELACIONES SINTÁCTICAS EN LA UNIDAD DOMÉSTICA PATRIMONIAL EN LOS OASIS DE SAN PEDRO DE ATACAMA}

Desde la disciplina de la arquitectura los estudios referidos a la vivienda patrimonial de San Pedro de Atacama se han enmarcado en el análisis de una tipología bastante homogénea, la cual rescata una tradición local y de construcción en barro cuyas calidades constructivas y espaciales son excepcionales. En este contexto "Espacios Intermedios: Respuesta arquitectónica al medio ambiente" (Kapstein, 1988), "Estudio de la Vivienda Atacameña" (Gutiérrez \& Maizares, 2001), "Levantamiento arquitectónico de la vivienda vernácula en San Pedro de Atacama" (Serrano, 2017), articulan un discurso que relaciona una serie de casos de arquitectura doméstica de gran valor patrimonial en esta localidad.

La tipología referida como vivienda típica y patrimonial atacameña, corresponde a la expresión de una arquitectura doméstica, propia y particular, presente en el poblado de San Pedro de Atacama y en sus "ayllús" adyacentes (Serrano, 2017). Corresponden a construcciones realizadas en adobes, adobones y excepcionalmente en piedra, cuya conformación final sería el resultado de múltiples eventos de ampliaciones, adhesiones y reconstrucciones,

6. Imagen exteriores vivienda familia León. Registro propio (2009).

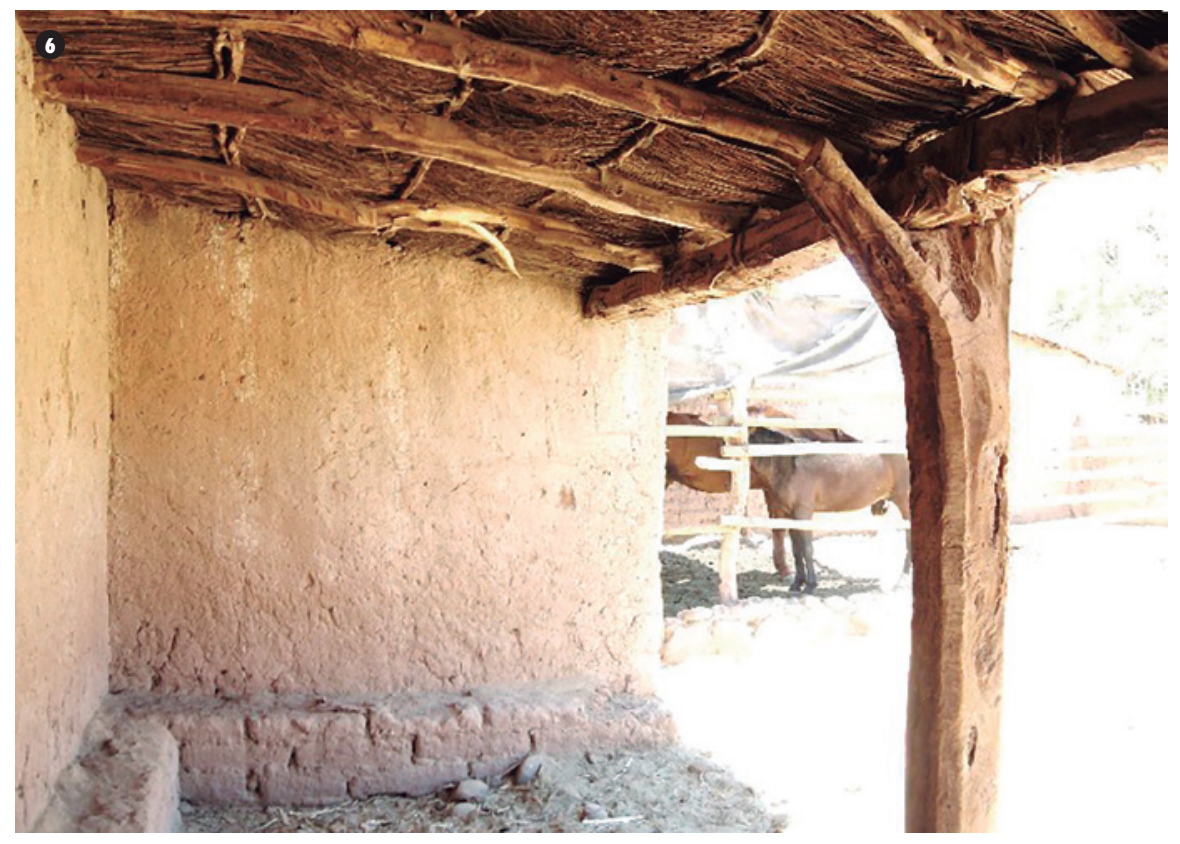

en algunos casos construidas en períodos que abarcarían varias generaciones.

Si bien aún no se realizan estudios que nos puedan otorgar una línea cronología precisa de estas construcciones patrimoniales, se estima que los recintos de mejor factura se realizarían entre las últimas décadas del siglo XIX hasta 1930 "época gloriosa de los toros". Período en el cual el oasis de San Pedro de Atacama se consolida cumpliendo un rol estratégico, como "nodo fundamental", en la articulación regional del tráfico ganadero proveniente de Argentina (FIGURA 6).

"El motor de dicha transformación fue la instalación de grandes industrias en la región: el mineral de plata de Caracoles, los cantones salitreros de Pampa Central y El Toco, y posteriormente, el yacimiento cuprífero de Chuquicamata" (Vilches, Sanhueza \& Garrido, 2014, p. 76).

Período de bonanza que finalizaría debido a la crisis económica mundial y de la industria del salitre, concluyendo de forma definitiva en 1948 debido a la finalización del proyecto ferroviario que unió las ciudades de Salta y Antofagasta, absorbiendo las ultimas remanencias de dicho tráfico ganadero. No obstante, algunos de los recintos construidos serían mucho anteriores a dicho período, evidenciado a través de los sistemas y materiales constrictivos utilizados en su construcción, como también, se aprecia la existencia de adiciones y reparaciones realizadas con posterioridad (FIGURA 7).

Los primeros cinco casos que se presentan a continuación corresponden a modelados esquemáticos realizados a partir de los trabajos de levantamiento planimétrico realizados por Glenda Kapstein (1988) y por Magdalena Gutiérrez y Roberto maizales (2001), el sexto caso corresponde a un registro y levantamiento propio (2009). Lamentablemente muchas de estas residencias ya no existen debido, principalmente, a las incontables demoliciones que se han sucedido en San Pedro de Atacama en relación a una alta demanda turística que requiere de nuevos servicios que ha dado paso a un progreso, hasta el momento, bastante mal entendido (FIGURAs 8, 9 y 10). 
7. Imagen de zaguán y el horcón, vivienda familia León. Registro propio (2009).

8. (Izquierda) (1) Casa Sra. Iris Hidalgo, Calle Tocopilla SPA. (Derecha) (2) Casa de los Ancianos, Calle Tocopilla SPA. Modelado digital y esquema funcional a partir de planimetría preexistente; (1) G. Kapstein, 1988; (2) Magdalena Gutiérrez y Roberto maizales, 2001. Elaboración propia (2012).

9. (Izquierda) Casa del Pukara 1, Ayllu Quitor OSPA. (Derecha) (2) Casa Sr. Tijerina, Ayllu Sequitor OSPA. Modelado digital y esquema funcional a partir de planimetría preexistente; (1) G. Kapstein, 1988; (2) Magdalena Gutiérrez y Roberto maizales, 2001. Elaboración propia (2012).
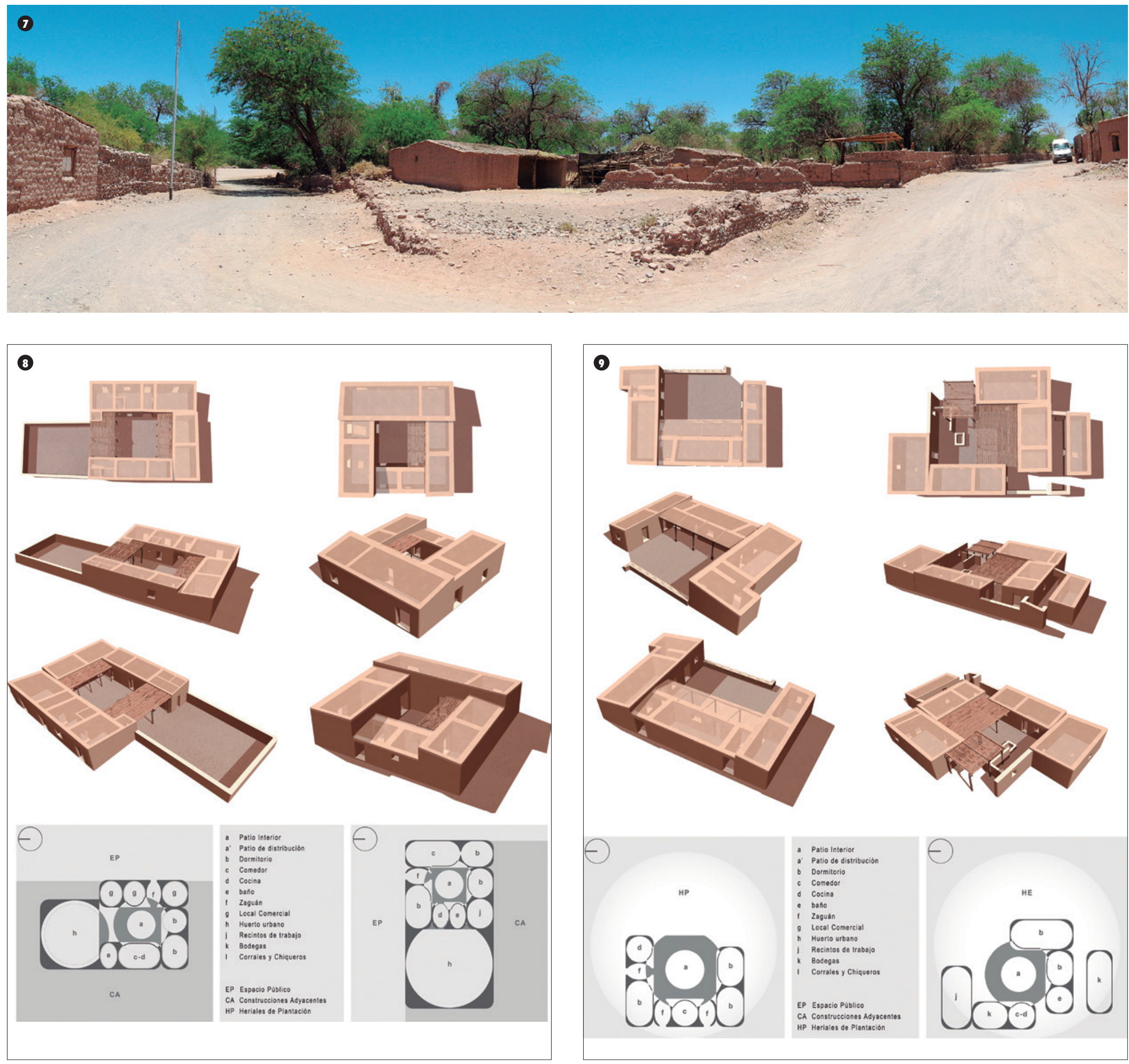
10. (Izquierda) Casa del Pukara 1, Ayllu Quitor OSPA. (Derecha) (2) Casa Sr. Tijerina, Ayllu Sequitor OSPA. Modelado digital y esquema funcional a partir de planimetría preexistente; (1) G. Kapstein, 1988; (2) Magdalena Gutiérrez y Roberto maizales, 2001. Elaboración propia (2012).

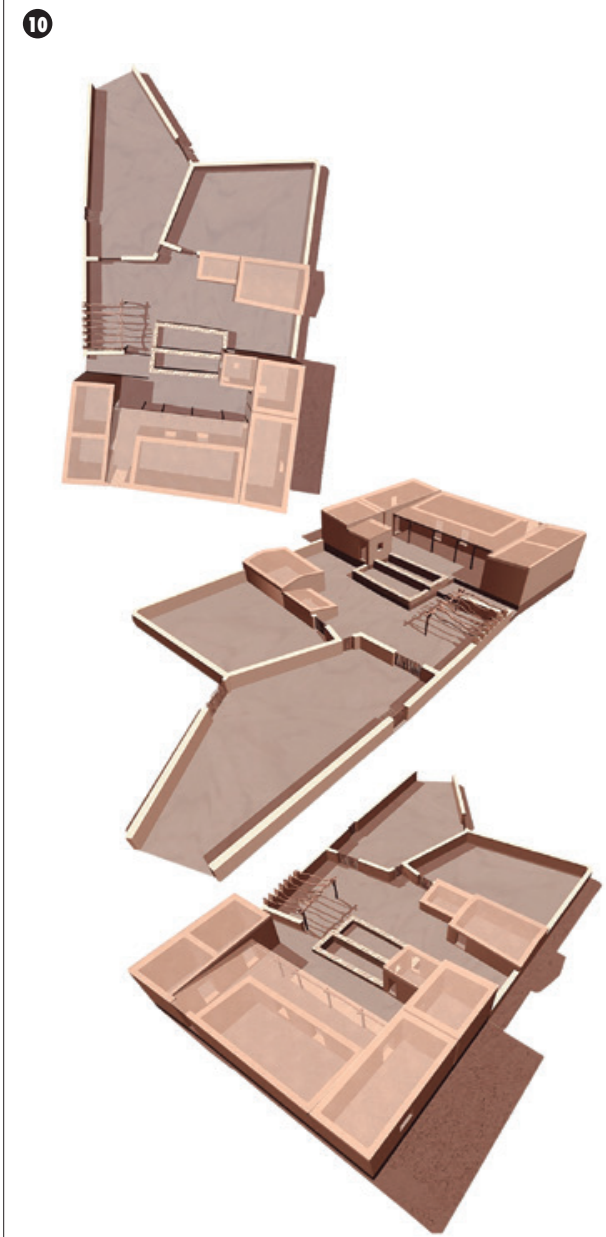

-
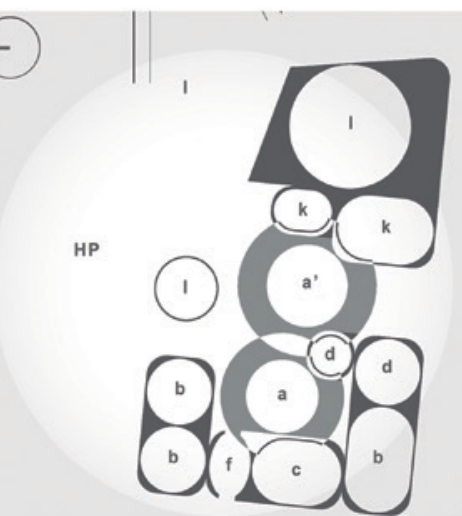

a Patio Interior

a' Patio de distribución

b Dormitorio

Comedor

d cocina

i baño

g. Local Comercial

huerto urbano

Recintos de trabajo

B Bodegas

Corrales y Chiqueros

EP Espacio Público

CA Construcciones Adyacentes

HP Heriales de Plantación
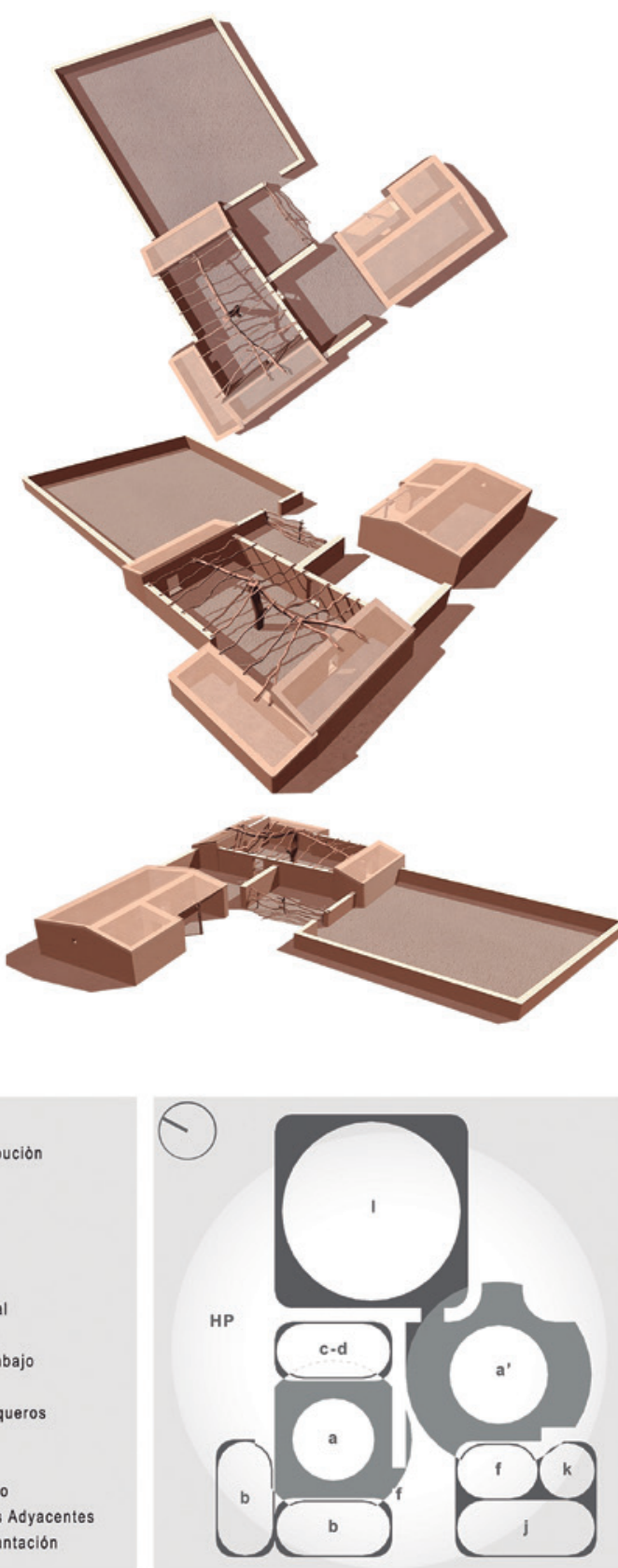

En todos los casos expuestos la construcción de los recintos que constituyen la unidad doméstica se organiza en torno a un patio central jerárquico, enmarcando la primera intención de planificación de las viviendas. Robert Maizares arquitecto y comunero de la localidad, en entrevista audiovisual realizada como parte del proyecto de investigación "Levantamiento arquitectónico y cultura constructiva de la vivienda vernácula en San Pedro de Atacama" (Serrano, 2017), relaciona esta intencionalidad con la importancia esencial que tienen las festividades del carnaval para la comunidad atacameña, ocasión donde la vivienda se hace pública acogiendo a la festividad y a sus celebrantes. Sin embargo, también cobra relevancia en otros aspectos esenciales de integración comunitaria, firmemente implícitos en el ideario colectivo; relacionando aspectos sagrados, a través de la realización de actividades como ofrendas a la tierra, fiestas comunales, celebraciones de matrimonios y funerales; con aquellos más domésticos y cotidianos, al ser la espacialidad donde se situaban los hornos de barro para la cocción de algunos alimentos, el almacenaje esporádico de bienes y el lugar donde se ejecutan algunos trabajos en relación de los oficios de quienes integran estas unidades domésticas.

Otro aspecto que caracteriza a estos patios son las cubiertas incorporadas con el fin de conseguir un control lumínico y climático adecuado, proporcionando las sombras necesarias para que las permanencias se sucedan. En algunos casos de modos puntuales mientras en otros cubriendo completamente dicha espacialidad. Estas estructuras fueron realizadas a través de vigas y horcones de chañar, como elementos soportantes, a los cuales se les incorporaron tamices livianos de cañas o atados de ramas secas. Como una variante más consolidada, se elaboraron estructuras porticadas a través de horcones labrados de chañar soportando la prolongación, de una o más de una, de las cubiertas de los recintos que componen el conjunto arquitectónico. 
Por otra parte, aquellos volúmenes construidos que se organizan en torno al patio central, delimitándolo y consolidándolo, corresponden a construcciones de adobes o adobones de planta rectangular con divisiones interiores, cubierta a una o dos aguas construidas mediante vigas de chañar, cielo de caña o brea, y torta de barro. Las dimensiones de estas construcciones variarían en relación al uso particular de cada una de ellas como, también, debido a los distintos períodos en que fueron construidas, por lo cual existe una diversificación considerable entre el tamaño de los adobes utilizados y la evidente variación en las técnicas constructivas en los diferentes "volúmenes construidos" dentro de una misma unidad doméstica.

De los casos presentados, aquellos ubicados dentro del casco urbano, se diferencian respecto a los existentes en los sectores agrícolas básicamente debido a las limitantes y condicionantes que la trama urbana impone, reflejado en un mayor grado de cerramiento del conjunto arquitectónico al situar los volúmenes construidos en los límites perimetrales separando, con ello, el espacio correspondiente al patio central de su contexto inmediato (espacio público y construcciones adyacentes). En algunos casos estas residencias urbanas vinculan pequeños huertos o jardines a través de la anexión de un solar contiguo donde predominan las plantaciones de árboles frutales.

La idea de patio posterior configurado y compartido por tres o cuatro viviendas se replica en los pueblos del altiplano. La linealidad propia de los pueblos de la precordillera da paso a tejidos más irregulares o tendientes a la cuadrícula pero con la identificación de estas células habitacionales (M. Hurtado, 2012, p. 38).

En los casos donde la vivienda se emplaza en sectores agrícolas o "ayllús", en terrenos que alcanzan varias hectáreas ocupadas principalmente como eriales de plantación (maizales, hortalizas y alfalfas), existe una mayor libertad formal y una clara intencionalidad en la generación de relaciones contextuales, vinculando la orientación del conjunto arquitectónico hacia la luz de la mañana y el volcán tutelar LikanKabur (cardinal este).

En este contexto, algunas de las residencias ubicadas en los "ayllús" presentan situaciones aún más diversas que complejizan el ordenamiento de la unidad doméstica, principalmente, debido a la anexión de programas complementarios en una estructura funcional mayor, principalmente, vinculando el manejo agroganadero y estanciero. Dentro de esta complejidad se integran construcciones como corrales, chiqueros, establos, bodegas para el forraje y talleres, que son organizadas a través de patios secundarios que cobran relevancia como nuevos espacios de mediación y distribución en función del ordenamiento de la complejidad descrita.

\section{DISCUSIÓN}

De las relaciones sintácticas presentes en la unidad doméstica vernácula y patrimonial, existente en San Pedo de Atacama y sus contextos próximos, es posible mencionar que la invariable que reaparece en la totalidad de los casos expuestos tiene relación con el orden preestablecido de los recintos construidos, siempre en función de la apropiación y posterior consolidación de un vacío central y jerárquico que constituye el núcleo fundamental de la unidad doméstica.

En la arquitectura de los períodos formativos tempranos esta relación surge a través de los primeros corrales, espacialidades portadoras de un firme valor ideológico implícito al representar los aspectos más sagrados de la comunidad, relacionados principalmente con la estabilidad de recursos. Para, posteriormente, tras la generación de asentamientos cada vez más nucleados y complejos, hacia el formativo medio, consolidar su organización a través de la construcción de plazas públicas y espacialidades intermedias. Estas últimas, altamente prácticas al albergar la mayor parte de las actividades domésticas de interacción social y de trabajo participativo.

Este fundamento trascendental, presente ya desde las primeras manifestaciones de la arquitectura doméstica, es también el sustento de la vivienda patrimonial de fines del siglo XIX e inicios del XX representado en sus patios centrales. En ellos se recoge la síntesis simbólica y pragmática de los primeros corrales, pero también de los espacios intermedios del formativo tardío. En estos patios se integran los aspectos sagrados con los de participación comunitaria y labores domésticas. Es aquí donde se realizaban y se siguen realizando actividades que sacralizan esta porción de desierto contenido, ya sea a través de celebraciones, oraciones, ofrendas y pagos a la tierra; en comunión con los usos más cotidianos a los que sirve, especialmente debido a la multiplicidad de manifestaciones sociales y de trabajos participativos.

Esta espacialidad que pareciese ser remanente de la tradición constructiva más antigua que se conoce del territorio en estudio podría vincularse, también, al hecho que gran parte de las actividades domésticas y comunitarias pueden ser desarrolladas, prácticamente durante todo el año, en exteriores mínimamente controlados "invariable climática sobre la cual se articula toda esta arquitectura, definiendo claramente los espacios protagónicos desde las primeras manifestaciones" (Rojas, 2014, p. 71).

Otro aspecto fundamental corresponde a la disposición de los volúmenes construidos, recintos modulares altamente interiorizados y muy controlados, en torno a la espacialidad antes descrita. Desde un planteamiento diacrónico, en el período formativo estos recintos serían ocupados principalmente para el resguardo y para aquellas actividades más íntimas, mientras que hoy es evidente que las necesidades propias de la vida contemporánea demandan de interiores controlados para prácticamente la totalidad de las actividades 
domésticas, desembocado en una irreversible sustitución de patrones arquitectónicos que conlleva a drásticos cambios en los modos como concebimos y articulamos el espacio residencial. Sin embargo, en los casos referenciados correspondientes a las viviendas patrimoniales, algunas de ellas aún en existencia y uso, estos patrones se supeditan a órdenes propiamente locales y jerárquicos de esta arquitectura propiamente vernácula. Interiorizando aquellas actividades que requieren mayores grados de privacidad y de control climático en los "volúmenes construidos" y exteriorizando otros aspectos menos restrictivos en la espacialidad central, altamente versátil, correspondiente al patio central.

Un último aspecto de importancia relaciona una mayor complejidad, pero también una mayor libertad formal, existente en las viviendas ubicadas en los sectores agrícolas ("ayllús"), donde es evidente la intencionalidad de orientar el conjunto arquitectónico jerarquizando las relaciones visuales y aperturas del espacio construido hacia la luz de la mañana y el volcán protector LikanKabur. Como también, articulan una complejidad mayor, debido a sus programas anexos, a través de la generación de patios secundarios ordenando su compleja funcionalidad programática.

Sin duda, tanto los asentamientos arqueológicos como las viviendas patrimoniales, a la vez de representar uno de los mayores valores materiales que aún persisten en estas zonas, establecen órdenes propiamente locales arraigados en un bagaje cultural, arquitectónico, y social, cimentado de manera "nueva" sobre el aprendizaje que deja cada estrato anterior.

Sin duda, entender el panorama actual del oasis de San Pedro de Atacama como parte de este proceso en vez de entenderlo como un fin en sí, nos otorga pautas teóricas posible de ser integradas como estrategias de diseño al abordar procesos de planificación, sobre todo, en la generación de nuevas propuestas arquitectónicas, que aun siendo contemporáneas, integren relaciones sintácticas en una lógica de procesos sustentados en los aspectos fundamentales de aquella arquitectura arraigada en un patrimonio social, material e inmaterial, en existencia y en evidente evolución.

\section{AGRADECIMIENTOS}

Quiero expresar mi gratitud a la comunidad atacameña, a todos quienes han abierto las puertas de sus casas y de sus corazones compartiendo su inmensa sabiduría. Agradecer, también, a aquellos académicos que directa o indirectamente han contribuido a la realización de este proyecto, especialmente a Magdalena Gutiérrez, Haroldo Horta y Cristian Games, por su disponibilidad y paciencia.

\section{REFERENCIAS BIBLIOGRÁFICAS}

Adán, L., \& Urbina, S. (2007). Arquitectura formativa en San Pedro de Atacama. Estudios Atacameños: Arqueología y Antropología Surandinas (34), 7-30. Obtenido de (ISSN 0718-1043).

Agüero, C., \& Uribe, M. (2011). Las sociedades Formativas de San Pedro de Atacama: Asentamiento, cronología y proceso. Estudios Atacameños, Arqueología y Antropología Surandinas (42), 53-78. Obtenido de (ISSN 0718-1043).

Gutiérrez, M., \& Maizares, R. (2001). Estudio de la Vivienda Atacameña. Informe del Ministerio de Vivienda y Urbanismo de la Región de Antofagasta (no publicado).

Hurtado Saldías, M. (2012). Aportaciones andinas a la construcción de una identidad local. Revista 180, UDP Facultad de arquitectura, arte y diseño, 66.

Kapstein, G. (1988). Espacios Intermedios, respuesta arquitectónica al medio ambiente. Reedición, Revista ARQ (2016). Obtenido de (ISBN: $978-$ 956-9571-17-6).

Nuñez, L. (1992). Cultura y conflicto en los oasis de San Pedro de Atacama. Santiago: Universitaria.

Rojas, V. (2014). Reconstrucción de los ámbitos domésticos en San Pedro de Atacama. Antofagasta: Memoria de Título.

Serrano, M. (2017). Levantamiento arquitectónico y cultura constructiva de la vivienda vernácula en San Pedro de Atacama. Obtenido de www. pangeafundacion.org

Vilches, F., Sanhueza, L., \& Garrido, C. (2014). Arquitectura de remeseros en San Pedro de Atacama. ARQ, 76-85. Obtenido de (ISSN 0717 6996). 